\title{
A Model for Magnetic Reconnection
}

E. Allen Adler and RusselI M. Kulsrud

Plasma Physics Iraboratory, Princeton University

$$
\text { Princeton, New Jersey } 08540
$$

We model a forced reconnection roblem by two infinite wires that are erabedded in a plasma which carry parallel currents. They are broight together at a specified rate. The distance between the wires is taken as $2 \mathrm{a}\left(1-\mathrm{e}^{\mathrm{wt}}\right)$. For sma 11 displacements, the hydromagnetic equations can be linea-ized and solved asymptotically. For larger displic zements, the plasma behavior can be estimated by use $G_{1}$ scaling arguments. We determine a local velocity of magnetic reconnection and show that it is essentially equal to the maximum possible reconnection velocity (that of the corresponding vacuum case) up to the time when this velocity approaches the local Alfvér speed. We compare the details of our solution with the sweetParker and the Petschek reconnection thecries. $1,2,3$ 


\section{INTRODUCTION}

The process of magnetic reconnection at an $x$ point has been studied by plasma physicists for a long time. Ore question that has remained unsolved is: What is the maximum rate of reconnection? For reconnection to take place, resistive diffusion of the field lines must occur at the $x$ point. Resistive effects, however, need only be important in a small region near the $x$ point. The rate of reconnection is related to the rate of magnetic diffusion across this small region. The approach to reconnection problems is to study the resistive behavior in the vicinity of the $x$ point and then to match the fields and flows to those occurring away from the resistive region.

One phenomenon involving reconnection which is fairly well understood is the linear tearing mode. 4 Waddell, et al. 5 have shown that for the $m=1, n=1$ tearing mode in a cylinder, the growth rate of $\mathrm{s}^{-1 / 3}$ continues until saturation at finite amplitude. $\mathbf{s}^{-1}$ is the ratio of the resistive diffusion velocity to the Alfvén velocity. However, since this is an eigenmode, one would expect that faster reconnection rates could occur if there were an external driving force.

Attempts to estimate the maximum reconnection rate in the presence of external forces have been made by many people. Most of the arguments are based on those presented by Sweet ${ }^{1}$ and Parker ${ }^{2}$ or by Petschek. ${ }^{3}$ other authors have extended and elaborated on these ideas. Their work was reviewed recently by vasilyunas. 6 These authors have recognized that in a long, thin current layer, 
the major factor limiting the reconnection rate is the velocity of the matter leaving the current region. It was pointed out that the outflowing velocity can not exceed the Alfven speed taken immediately outside the layer. Sweet and Parker have assumed that the incoming velocity was related to the outgoing velocity by $\mathrm{v}_{\text {out }} \approx \mathrm{L} \delta^{-1} \mathrm{v}_{\text {in }}$, where $\mathrm{L}$ and $\delta$ are the length and width respectively of the current channel. Petschek argued that if $\delta$ is not a constant, but a function of distance taken lengthwise along the current channel, then the reconnection speed can approach the Alfvén speed divided by a logarithmic factor. In this paper we study the forced reconnection in the case where the field lines fan out in such a way that $v_{\text {out }} \approx v_{\text {in }}$. We can, then, test the hypothesis that reconnection can proceed at a rate such that the outgoing velocity is given by the Alfvern speed. To do this we look at an $x$ point formed between two current carrying wires. Reconnection is forced by bringing the two wires together. For small displacements of the wires, we can linearize the hydro-magnetic equations. This allows us to treat the problem in fair detail. Because of this treatment we can see the relationships among the reconnection rate, the forcing agent, and the resistivity. Also, the solutions obtained are compared to what is expected in Petschek's theory. This will give an insight into the effects of a driving force and external boundary conditions which are more realistic than those assumed by Petschek. Secondly, we can check some of Petschek's assertions by this comparison. Thus, we attempt to study the reconnection problem by considering a specific problem in detail. 
II. MODEL

In the problem considered, two wires, each carrying a fixed current in the same direction, are embedded in a plasma. (See Fig. 1.) The wires are brought together at an externally controlled rate. Initially, the magnetic field is taken to be the vacuum field of the two wires. The response of the plasma and field is then followed. Of particular interest is the comparison of the flux between the wire and the $x$ point to what it would be had the motion been executed in a vacuum.

Mathematically, the problem is studied in the framework of two-dimensional resistive MHD theory. The equations governing the model are:

$$
\begin{aligned}
& \frac{\partial \psi}{\partial t}+\vec{v} \cdot \nabla \psi=n j=n \nabla^{2} \psi, \\
& \rho \frac{d \vec{v}}{d t}=\vec{j} \times \vec{D}-\nabla P, \\
& \frac{\partial \rho}{\partial t}+\nabla \cdot(\rho \vec{v})=0,
\end{aligned}
$$

In these equations $\psi$ is a flux function defined such that $\hat{B}=\hat{z} \times \nabla \psi$, where $\hat{\mathbf{z}}$ is parallel to the wires. Equation (1) is found by integrating a version of Faraday's law in which the electric field is given by Ohm's law. In general this integration introduces a constant term. This constant may be eliminated, however, by a suitable choice of a point at which $\psi$ vanishes. It can be shown that if the wires are small and perfectly conducting (which is 
assumed to be the case), then choosing $\psi(r, t)$ to vanish at the centers of the wires will cause the aforementioned integration constant to vanish. Finally, the above equations are made complete by the addition of an equation of state. In this paper we will assume that the resistivity $n$ is homogeneous in space and constant in time.

Boundary conditions must be supplied for the plasma bounciaries at the wire surfaces and at infinity. We choose the following conditions :

$$
\begin{aligned}
& \psi \text { is continuous at the wire surface, , } \\
& \mathrm{v}=0 \text { at } \mathbf{r}=\mathrm{R},
\end{aligned}
$$

where $\mathrm{R}$ is large compared to any other length in the problem. Note that Eq. (4) is equivalent to the assumption that any surface current on the wire surface is integrable.

Two assumptions are made which allow simplification. These assumptions are usually made in considering problems of this type.

$$
\begin{aligned}
& \frac{\mathrm{n}}{\mathrm{a}} \ll \mathrm{v}_{\text {wire }} \\
& \mathrm{v}_{\text {wire }} \ll \mathrm{v}_{\text {Alfvén }} .
\end{aligned}
$$

In EG. (6) a is a macroscopic length scale for the magnetic field [cf Eq. (8)]. The first assumption allows the plasma to be treated as perfectly conducting except in a small region near the $x$ point. 
The second assumption allows inertia to be neglected except near the $x$ point.

Finally, the wire displacement as a function of time must be chosen. The positions of the wire centers are chosen to have the following time dependence:

$$
r_{\text {wire }}= \pm a\left(1-e^{\omega t}\right)
$$

where the problem wil.l be studied for values of $t$ in the interval $(-\infty, 0)$. This choice of the wire displacement allows us to study the effects of small displacements (i.e., $|\omega t|>1$ ) with the consequences of larger displacements appearing gradually (as wt $\rightarrow 0$ ). Note that regardless of the value of $\omega$, for $|t|$ sufficiently large, the displacement is small; whereas for $\omega$ small, the velocity is always small. Hence, Eq. (7) may be regarded as a condition on $\omega$.

\section{LINEAR THEORY}

When the wire displacement is small, it is valid to linearize the equations. It is helpful to introduce an orthogonal coordinate system in which one coordinate is the zero-order flux (see Appendix). These coordinates are:

$$
\begin{aligned}
& \Psi=\left\{\left[(x+a)^{2}+y^{2}\right]\left[(x-a)^{2}+y^{2}\right]\right\}^{1 / 2} \\
& \Phi=\tan ^{-1}\left[2 x y\left(x^{2}-y^{2}-a^{2}\right)^{-1}\right] .
\end{aligned}
$$


In this coordinate system, the linearized version of Eq. (1) becomes:

$$
\omega \psi+4 I_{0} \Psi^{-1}\left(\Psi^{2}+2 a^{2} \Psi \cos \Phi+a^{4}\right)^{1 / 4} v_{\Psi}=n j
$$

We now proceed in the following way. First, we analyze the problem everywhere except in the vicinity of the $x$ point. Then an analysis is done of the behavior around the $x$ point. Finally, the results are put together in a consistent way.

\section{A. External Region}

The assumption in Eq. (7) implies that inertia is negligible except in the vicinity of the $x$ point. Because of this, Eq. (2) reduces to the equation of two-dimensional magnetostatics.

$$
\vec{B} \cdot \nabla j=0 \Rightarrow j=j(\Psi)
$$

Further simplification can be made by assuming that the speed of sound, $\mathrm{c}_{\mathbf{s}}$, is much smaller than the Alfvên speed. This means that the pressure can be neglected. This assumption, put together with Eq. (12), implies that the field is force free and, therefore, current free. The equations reduce, except at the $x$ point, to:

$$
\nabla^{2} \psi=0
$$

where $\psi$ is the change in the flux from what it had been before the wire motion was begun. This equation can be extended to include 
the effects of the current at the $x$ point. This can be done if the singular region around the $x$ point is small, then the dominant effect of the current at the $x$ point on the external field will come from its lowest nonvanishing nultipole moment. It is now assumed, subject to a postieri verification that the lowest moment is the dipole moment. Therefore, an equation, valid in the exterior region, which includes the effects of the current near the $x$ point, is (see Appendix):

$$
\frac{\partial^{2} \psi}{\partial \Psi^{2}}+\frac{1}{\Psi} \frac{\partial \psi}{\partial \Psi}+\frac{1}{\psi^{2}} \frac{\partial^{2} \psi}{\partial \Phi^{2}}=\frac{I}{a^{2}} \delta\left(\Psi-a^{2}\right) \delta(\Phi-\pi)
$$

where $I$ is given by a solution to the region around the $x$ point. Next, we derive boundary conditions on the perturbed flux, $\psi$, from conditions in Eqs. (4) and (5). First, the wire surface is considered. Assume that the wire is shaped so that its outer surface is given by $\Psi=\varepsilon_{w}$. (For $\varepsilon_{w}$ small this means only a small deviation from a circular cross-section for the wire.) The boundary condition on $\psi$ is $\left.\frac{\partial \psi}{\partial t}\right|_{\text {wire }}=\left.\frac{\partial \psi}{\partial t}\right|_{\text {plasma }}$. This may be expressed in a more convenient form using the facts that the wires are perfectly conducting and that the equations are being linearized. We obtain $\left.\frac{\partial \psi}{\partial t}\right|_{\text {wire }}=-\left.v \cdot \nabla \psi_{0}\right|_{\text {wire }}=\mp I_{0} v_{0} \Psi^{-2} \frac{\partial \Psi}{\partial x}$, where $v_{0}$ is the wire velocity $\mp a w e^{\omega t}$, and $I_{0}$ is the current in each of the wires. When $(\partial \Psi / \partial x)$ is expanded for $\Psi$ small, the following condition is obtained:

$$
\psi_{\Psi=\varepsilon_{W}}=4 I_{0} v_{0} \omega^{-1}\left[a \varepsilon_{W}^{-1} \cos \Phi+(2 a)^{-1}+0\left(\varepsilon_{W}\right)\right]
$$


The second boundary condition is simply $\left.\psi\right|_{r=R}=0$. For convenience we assume that $a^{-2} R^{2}>a^{2} \varepsilon_{w}^{-1}$.

The problem is thus reduced to a standard boundary value problem in cylindrical coordinates. To lowest order in $(1 / R)$ and $\varepsilon_{w}$, the solution in the vicinity of the $x$ point is:

$$
\begin{gathered}
\psi=-2 a^{-1}{ }^{-1} I_{0} v_{0}-\pi^{-1} I \log a\left[2-\log \varepsilon_{W}(2 \log a)^{-1}\right] \\
+\pi^{-1} I \log r
\end{gathered}
$$

This equation must be matched to the interior solution. Note the origin of the three terms. The first term is the flux change that that would occur if $I=0$, i.e., if there were no plasma. This is due directly to the decrease in distance between the two wires. Thus, the first term represents the flux change in the case of perfect reconnection. The third term represents the flux accumulation due to the current at the $\mathrm{x}$ point and is, therefore, a measure of the amount of unreconnected flux. The middle term is due to the image currents induced in the wires by the currents at the $x$ point. In a true three dimensional situation, one would expect this term to become negligible. (The flux produced by image currents at $r=R$ is $0\left(R^{-1}\right)$ and hence negligible.)

\section{B. Interior Region}

Near the $x$ point, neither inertia nor resistivity can be neglected. Furthermore, since the Alfvén speed vanishes at the $x$ point, the neglect of pressure is no longer necessarily valid. The relevant 
comparison here is between the sound speed and the local Alfven speed, $(\delta / a) v_{a}$, where $\delta$ is the size of the singular region. We will consider two opposite limiting cases. First, we will assume that the sound speed is sufficiently small that the pressure may be neglecter, even in the interior region. In the second case, it is assumed that $c_{s} \gg(\delta / a) v_{a}$ and, hence, that the interior region is incompressible.

1. Pressureless case $\left[c_{s} \ll(\delta / a) v_{a}\right]$

The equations here are Eqs. (11) and (2) which, when linearized, can be reduced to the following:

$$
v_{\Psi}=-4 I_{0} \rho_{0}^{-1} \omega^{-1} \Psi^{-1}\left(\Psi^{2}+2 a^{2} \Psi \cos \Phi+a^{4}\right)^{1 / 4} j .
$$

Equations (17) and (11) can then be combined. Returning to polar coordinates, the result is:

$$
\left.\nabla^{2} \psi[\eta)^{-1}+r^{2} \varepsilon^{-2}\left(1-2 r^{2} a^{-2} \cos 2 \theta+a^{-4} r^{4}\right)^{-1}\right]=\psi
$$

where $\varepsilon^{2}=4^{-1} I_{0}^{-2} \rho \omega^{2} a^{4}$. In the interior region $r \ll a$, making this approximation we obtain:

$$
\nabla^{2} \psi=\varepsilon^{2} \psi\left(r^{2}+\eta \omega^{-1} \varepsilon^{2}\right)^{-1}
$$

Introducing a scaling $r=\varepsilon \lambda r^{\prime}$ where $\lambda^{2}=n \omega^{-1}$ this becomes (suppresising primes for convenience)

$$
\nabla^{2} \psi=\varepsilon^{2} \psi\left(r^{2}+1\right)^{-1}
$$

from this it can be seen that $\varepsilon \lambda$ is the scale size of the resistive 
region. Equation (20) may be solved by expansion using the fact that $\varepsilon^{2} \ll 1$. The result is

$$
\psi(r)=\psi(0)+\varepsilon^{2} \frac{\psi(0)}{2} \int_{0}^{r} \frac{\log \left(I+r^{2}\right)}{r} d r+0\left(\varepsilon^{4}\right) .
$$

The expansion procedure is valid as long as $\log \left(r \varepsilon^{-1} \lambda^{-1}\right) \ll \varepsilon^{-1}$. Note that taking this expansion to first order in $\varepsilon^{2}$ is equivalent to the constant $\%$ approximation as introduced by Furth, et al. 4

2. Incompressible case $\left[c_{s}>(\delta / a) v_{a}\right]$

The fluid equation of motion in this case may be derived by taking the curl of the Jinearized version of Eq. (2). If the result is then differentiated with respect to $\Phi$, and if incompressibility is used, the following equation can be obtained:

$$
\nabla^{2} u=16 \rho_{0}^{-1} \omega^{-1} \psi^{-2} I_{0}\left(\Psi^{2}+2 a^{2} \Psi \cos \Phi+a^{4}\right)^{1 / 2} \frac{\partial^{2} j}{\partial \Phi^{2}}
$$

where $u=\left({ }^{2}+2 a^{2} \Psi \cos \Phi\right)^{-1 / 4} \Psi v_{\Psi}$. Next, define $w=\omega^{-1} I_{0} u$, and Eqs. (12) and (24) can be written in a simplified form.

$$
\begin{aligned}
& w=4^{-1} \psi^{2}\left(\Psi^{2}+2 a^{2} \Psi \cos \Phi+a^{4}\right)^{-1 / 2}\left(\lambda^{2} j-\psi\right), \\
& \Psi \frac{\partial}{\partial \Psi}\left(\Psi \frac{\partial w}{\partial \Psi}\right)+\frac{\partial^{2} w}{\partial \Phi^{2}}=a^{4} \varepsilon^{-2} \frac{\partial^{2} i}{\partial \Phi^{2}} .
\end{aligned}
$$

Due to the nonseparability of these equations, we have been unable to find an analytic solution. Nevertheless, the gross scaling of the interior region may be obtained from these equations. From Eq. (24) one finds that $w \approx \varepsilon^{-2} a^{4} j$; while from Eq. (23) it 
follows that $w \approx r_{5}^{-2} a^{4} \lambda^{2} j$ where $r_{s}$ is the scale size of the resistive region. Comparison of these indicates that $r_{s} \approx \varepsilon \lambda$.

Before we can calculate the flux due to this current, we must deal with the fact that the current in a small layer lying along the separatrix does not vanish $h$ ith increasing distance from the point. In fact, due to incompressibility and to the fact that the lines converge toward the separatrix, $\eta_{1}$ and, hence, the vorticity, must increase with increasing distance from the $x$ point. Thus, there will be four current sheets eminating from the $x$ point along the separatrix. Note that the effect will be cut off at a distance where $\eta_{1} \approx c_{s}$. The important point, however, is that the current in each of the four sheets has the same sign. Therefore, due to symmetry, these currents will make no contribution to the field perpendicular to the line between the two wires along that line. Hence, these currents will not contribute to the flux at the $x$ point. Because of this, we may estimate the flux at the $x$ point due to the current there as $\psi \approx r_{\mathbf{s}}^{2} j \approx \lambda^{-2} \psi \varepsilon^{2} \lambda^{2} \approx \varepsilon^{2} \psi$. This result is similar to that for the pressureless case, i.e., negligible flux build up.

\section{c. Matching}

The entire solution to the linearized problem may be completed by matching the external solution (Sec. A) to the interior (Sec. B). This may he done explicitly in the pressureless case. First, the interior solution [Eq. (2I)] is expanded asymptotically for large $\mathbf{r}$, and then the scaled variables are replaced by the original variables. The result is: 


$$
\begin{aligned}
\psi(r)=\psi(0)+\varepsilon^{2} \psi(0)[ & \left.\frac{\pi^{2}}{24}+\frac{1}{2}(\log \varepsilon \lambda)^{2}\right]-\varepsilon^{2} \psi(0) \log \varepsilon \lambda \log r \\
& +\frac{\varepsilon^{2} \psi(0)}{2}(\log r)^{2}+C\left(\varepsilon^{-2}\right)+O\left(\varepsilon^{4}\right) .
\end{aligned}
$$

Note that for values of $r$ far outside of the singular region, $|\log \varepsilon \lambda|>|\log x|$ and, hence, the last cerm may be neglected compared to the penultimate term. This equation may now be matched directly with Eq. (16). It is then seen that:

$$
\begin{aligned}
& \psi(0)=-\frac{2 I_{0} v_{0}}{a w}\left\{1-\varepsilon^{2}\left[\frac{\pi^{2}}{24}+\frac{(\log \varepsilon \lambda)^{2}}{2}-\log \varepsilon \lambda \log a\left(2-\frac{\log E_{w}}{2 \log a}\right)\right]\right. \\
& \left.+O\left(\varepsilon^{4}\right)\right\} \\
& I=\frac{2 \pi \varepsilon^{2} I_{0} V_{0}}{a \omega} \log \varepsilon \lambda .
\end{aligned}
$$

Note that since $I<0$, it froduces a magnetic field which is in the same direction as the vacuum field at points along the line connecting the wire centers. Thus, because of this current, the amount of flux that is reconnected is smaller than that in the vacuum case. The amount of reconnected flux at a time $t$ is given by the difference $\psi(0,0)-\psi(0, t)$. We are interested in comparing the amount of reconnected flux when the wires are moved in a plasma to that when they are moved in a vacuum. We find from Eq. (26) that:

$$
\psi(0, t)-\psi_{\operatorname{vac}}(0, t)=\frac{\varepsilon^{2} I_{0} v_{0}}{a \omega}\left[\frac{\pi^{2}}{12}+(\log \varepsilon \lambda)^{2}-2 \log \varepsilon \lambda \log a\left(2-\frac{\log \varepsilon_{w}}{2 \log d}\right] .\right.
$$


Thus, the flux buildup is negligible as long as $\varepsilon \ll 1$. Note also that the flux buildup depends upon the resistivity only logarithmically.

IV. NONLINEAR THEORY

Because of the small size of the inner region, the zero-order field is weak and the velocities are large so that the equations describing this region become nonlinear even when the wire displacement is still very small compared to a. Unfortunately, the nonIinear equations defy our attempts at analytic solution. In this section, we are able to present some crude estimates of the behavior in the incompressible case based on these nonlinear equations. (We feel that this is more general; because if the plasma were pressureless, then the $\vec{v} \cdot \nabla \vec{v}$ terms would lead to a shock that would heat the plasma near the $x$ point.)

\section{A. Onset of the Nonlinearity}

First, we try to estimate the size of the wire displacement at the point at which the nonlinear terms become appreciable. For the inertial terms, this implies a comparison of $(\partial \vec{v} / \partial t)$ with $\vec{v} \cdot \nabla \vec{v}$. We estimate $v_{1}$, the component of the velocity perpendicular to $B$, by taking its value in the external region, near to its boundary with the inner region. From Part III we know that the size of the inner region is $\varepsilon \lambda$. Thus,

$$
v_{1} \approx \omega \Psi(0) I_{0}^{-1} a^{2} \varepsilon^{-I} \lambda^{-1} \approx \omega \xi_{w} a(\varepsilon \lambda)^{-1}
$$


where $\psi(0)$ is given by Eq. (26) and $\omega^{-1} v_{0}$ has been written as $\xi_{w}$ ' the wire displacement. Furthermore, one expects that $\eta_{\|} \approx v_{\perp}$ and that $\nabla$ can be estimated by $(\varepsilon \lambda)^{-1}$. Using this and Eq. (29), the size of $\vec{v} \cdot \nabla \vec{v}$ may be estimated and compared to $(\partial \vec{v} / \hat{c} t)$.

$$
\left(\frac{\partial v}{\partial t}\right)^{-1} v \cdot \nabla v \approx \omega^{-1}(\varepsilon \lambda)^{-1} v_{\perp} \approx \xi_{w} a(\varepsilon \lambda)^{-2}
$$

Thus, when $\left(a^{-1} \xi_{\mathrm{w}}\right) \approx\left(\mathrm{a}^{-1} \varepsilon \lambda\right)^{2}$, the nonlinear inertia terms shoulid become appreciable. We may calculate the fluid displacement at the $\mathrm{x}$ point at this time.

$$
\xi_{x} \approx(E \lambda)^{-1} \text { a } \xi_{w} \approx E \lambda,
$$

therefore, the inertia becomes nonlinear when a fluid particle has passed through the singular region.

The next question is whether or not the magnetic field is nonlinear for $\left(a^{-1} \xi_{w}\right) \approx\left(a^{-1} \varepsilon \lambda\right)^{2}$. To determine this it is necessary to calculate the current at the $x$ point. Ics magnitude may be estimated from Ohm's law.

$$
j_{x} \approx w n{ }^{-1} \psi(0) \approx a^{-2} I_{0} \lambda^{-2} \xi_{w} a \approx E^{2} a^{-2} I_{0} .
$$

Thus, for $\varepsilon \ll 1$, terms involving the magnetic field may still be linearized.

B. Effects of Nonlinear Inertia

of primary interest, is the amount of flux that has accumulated between the wire center and the $x$ point (i.e., the amount of 
unreconnected ilux) due to the currents in the inner region. We assume that the inner region has dimensions $\delta$ and $B S$ (see Fig. 2), where it is expected that $\beta>1$. The accumulated flux may be estimated as

$$
\delta \psi \approx j_{x} \delta^{2}
$$

where $j_{x}$ is the current at the $x$ point. Until the flux accumulation becomes significant compared to the reconnected flux, $j_{x}$ is still given by Eq. (32). This is true because the electric field is directly related to the rate of change of the total flux. To estimate the size of the inner region when nonlinear inertial terms are important, we compare $j_{x}$ as estimated in EG. (32) to the current needed to drive the flows. Since the magnetic field terms may still be linearized, $v_{\perp}$ may be estimated by Eq. (29) with the replacement of $\varepsilon \lambda$ by $\delta$. Since the field lines are changed only slightly from the zero-order field, it is again true that $v_{\|} \approx v_{1}$. Estimating $\nabla$ by $\delta^{-1}$ we find

$$
\rho v \cdot \nabla(\hat{z} \cdot \nabla \times \vec{v}) \approx \rho \omega^{2} \delta^{-4} \xi_{w}^{2} a^{2}
$$

In estimating the driving torque, we make use of the fact that the field terms can be linearized.

$$
\text { 吉. } \nabla j=\left(a^{-2} I_{0} \delta\right)\left(\delta^{-1} j_{x}\right) \approx a^{-2} I_{0} j_{x} \text {, }
$$

thus, from the curl of the fluid equation of motion, $\left(\rho \frac{d \omega}{d t}=\right.$ 古 $\cdot \nabla j$ where $\omega=\hat{z} \cdot \nabla \times \vec{v}), j_{x}$ may be estimated as 


$$
j_{x} \approx I_{0}^{-1} \rho \omega^{2} a^{4} \delta^{-4} \xi_{w}^{2} \approx a^{-2} I_{0} \varepsilon^{2} \xi_{w}^{2} a^{2} \delta^{-4} .
$$

Comparing Eqs. (36) and (32) we find

$$
\delta \approx(\varepsilon \lambda)^{1 / 2}\left(a \xi_{w}\right)^{1 / 4}
$$

Thus, the width of the singular region grows as the one fourth power of the wire disp?.acement. The flux accumulation may be calculated as

$$
\delta \psi \approx\left(a^{-2} I_{0} \lambda^{-2} \xi_{w} a\right) \varepsilon \lambda\left(a \xi_{w}\right)^{1 / 2} \approx a^{-2} I_{0} \varepsilon \lambda^{-1}\left(a \xi_{w}\right)^{3 / 2} .
$$

This estimate is valid as long as magnetic field terms can be linearized; that is until $j_{x} \approx a^{-2} I_{0}$ or until $\xi_{w} a \approx \lambda^{2}$. At this time, the rate of flux accumulation is

$$
\frac{\partial}{\partial t}\left(\delta \psi_{a c c}\right) \approx \varepsilon \omega \lambda^{2} a^{-2} I_{0} .
$$

This should be compared to the rate of flux change due to the wire motion, which at this displacement is given by

$$
\frac{\partial \psi}{\partial t} \approx \omega a^{-1} I_{0} \xi_{w} \approx \omega a^{-\bar{z}} I_{0} \lambda^{\bar{z}}
$$

comparing Eqs. (39) and (40), it is seen that the flux pile up is still negligible as long as $\varepsilon \ll 1$. Thus, although nonlinear iner $j$ al effects cause flux to pile up at a faster rate than in the linear case, the rate of pile up is still negligible compared to the reconnection rate. 
Note that there is still a large current along the separatrix, as was encountered in the linear incompressible case. However, this current is anti-symmetric about the separatrix. Therefore, it makes no contribution to the field away from the separatrix and does not lead to any flux accumulation between the $x$ point and the wire.

c. Nonlinear Field and Inertia

As was remarked in the previous section, when the wire displacement reaches the size $\xi_{w} \approx a^{-1} \lambda^{2}$, the magnetic field in the inner region due to the currents there becomes equivalent in magnilude to the zern-order magnetic field. Along the $Y$ axis (see Fig. 2), the field due to these currents opposes the zero-order field. Thus, as the currents grow, additional $x$ points can appear. The current at these $x$ points would become equivalent to the current at the original $x$ point, since the electric field is nearly the same at all of these. points. Thus, there appears to be a tendency for the current layer to spread out along the $Y$ axis.

The length of the current layer may be estimated by assuming that the current distributes itself so that there is only one $x$ point. Since the zero-order field increases with distance for a long distance from the $x$ point and since the currents at the $x$ point are localized, it is clear that at some point the zero-order field will exceed the field $\vec{B}_{\ell}$ due to the local currents. Thus, in order to avoid an $x$ point, the zero-order field must never be less than the field due to tine local currents. This puts a condition on the derivative of the local field at the $x$ point. 


$$
\left|\frac{\partial B}{\partial y}\right|_{0} \mid \leqslant a^{-2} I_{0}
$$

using the Blot-Savart law we can write this condition in terms of the current.

$$
\left|-j(0)-\int d r d \theta r^{-1} j \cos 2 \theta\right| \lesssim a^{-2} I_{0}
$$

In several models mentioned here, $1,2,3$ the current is confined to a very small region around the $y$ axis and has the property that $\int r d \theta j(r, \theta) *$ constant. In these cases, the current layer can be approximated by a rectangular region of dimensions $\delta$ and $\beta \delta$. With a configuration of this type, one finds that in order to satisfy Eq. (41), $\beta \approx j_{X} a^{2} I_{0}^{-1}$. Thus, based on plausible assumptions concerning the nature of the current layer, its dimensions have been estimated.

Before one can estimate the amount of flux pile up for a given wire velocity, it is necessary to know the $y$ distance that a typical field line stays in the singular region. It is important to note that this distance may be much smaller than $\beta \delta$. It is needed in order to calculate $v_{\|}$from $v_{\perp}$. From previous sections of this paper as well as from Petschek's ${ }^{3}$ work, it can be seen that the maximum rate of reconnection depends critically upon the ratio $\mathrm{v}_{\|} / \mathrm{v}_{\perp}^{-1}$. The crudest estimate available, which assumes that the field is a superpostion of a rectangular current layer on top of the zero-order field, predicts that the field lines stay in the singular region for a distance $\beta^{1 / 2} \delta$ where $B \delta$ is the length of 
the current layer. This gives a maximum reconnection rate much faster than that of Sweet-Parker. ${ }^{1,2}$ Unfortunately, we have been unable to extend our investigation sufficiently to confirm or refute Petschek's ${ }^{3}$ claims of a maximum reconnection rate the order of the Alfvên speed.

\section{v. SUMMARY AND CONCLUSIONS}

We will now summarize the results of the previous two sections and attempt to point out what bearing they have on the general problem of reconnection at an $x$ point. In doing this it is useful to introduce the notion of a reconnection velocity. We define

$$
\left.v_{\text {reconnection }} \approx \frac{\partial \psi}{\partial t}\right|_{x}\left|\vec{B}_{\text {boundary }}\right|^{-1}
$$

where $(\partial \psi / \partial t)$ is evaluated at the $x$ point and $|B|$ is evaluated just outside the resistive region. It is of interest to compare $v_{\text {reconnection }}$ to the Alfuen speed in the external region. It is also

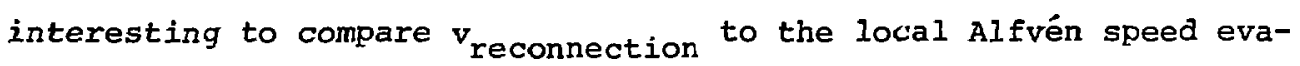
luated just outside the resistive region. In Fig. 3 we plot schematically the local Alfvên speed and the reconnection speed versus wire displacemer for the regimes discussad in Secs. III and IVB. It was shown in these sections that as long as $\varepsilon \ll 1$, almost complete reconnection occurs. From the graph, it can be seen that for $\xi_{w} \leq a^{-1} \lambda^{2}, v_{\text {reconnection }} \leqslant v_{a \text { local }}$ as long as $\varepsilon \ll 1$. Thus, in these two regimes, the limiting reconnection speed is the local Alfvén speed. 
In the situation discussed by Petschek, ${ }^{3} \mathrm{v}_{\mathrm{a}}$ and $\mathrm{v}_{\mathrm{a}}$ local are identical. Thus, his assertions concerning the relationship of $v_{a}$ to $v_{\text {reconnection }}$ must be interpreted, in this case, as concerning the relationship of $v_{\text {a local }}$ to $v_{\text {reconnection }}$. In the two regimes studied here, Petschek' $5^{5}$ conclusions seem valid. That is to say, the maximum value of $v_{\text {reconnection }}$ is almost the local Alfvén speed and not some fractional power of this speed.

The ensuing regime, discussed briefly in Sec. IVC, presents a situation that is even closer to that envisioned by Petschek. ${ }^{3}$ This is true since the field is determined primarily by local currents, and these currents are confined to a long, thin layer. An understanding of the behavior here could answer the question of whether or not the field lines can fan out themselves (i.e., if they are not forced to fan out by external currents). Thus, the further study of this problem could give more insight into the reconnection process.

In summary we believe that our model supports the rate of reconnection proposed by Petschek if one takes the Alfvén speed as that local to the $x$ point. The open question then is whether a situation can develop such that the local Alfvén speed equals the finite Alfvén speed away from the $x$ point as was surmised by Petschek.

\section{ACKNOWLEDGMENTS}

We are grateful to $P$. Kaw, C. Oberman, and $R$. White for helpful discussions.

This work was jointly supported by U.S. AFOSR Contract No. F44620-75-C-0037 and NSF Grant No. AST76-80800 and U. S. Department of Energy Contract No. EY-76-C-02-3073. 
APPENDIX - Flux Coordinates for the Two Wire System

The orthogonal flux coordinates are given in terms of rectangular coordirates by (see Fig. A1):

$$
\begin{aligned}
& \Psi^{2}=\left[(x+a)^{2}+y^{2}\right]\left[(x-a)^{2}+y^{2}\right], \\
& \tan \Phi=2 x y\left(x^{2}-y^{2}-a^{2}\right)^{-1} .
\end{aligned}
$$

The standard vector and scalar differential operators may be written in these coordinates using the following expression for the length of a segment.

$$
d s^{2}=4^{-1}\left(\Psi^{2}+2 a^{2} \Psi \cos \Phi+a^{4}\right)^{-1 / 2}\left(d \Psi^{2}+\Psi^{2} d \Phi^{2}\right) .
$$

Several relationships between these coordinates and polar coordinates are:

$$
\begin{aligned}
& r=\left(\Psi^{2}+2 a^{2} \Psi \cos \Phi+a^{4}\right)^{1 / 4}, \\
& \Psi^{2}=r^{4}+a^{4}-2 a^{2} r^{2} \cos 2 \theta .
\end{aligned}
$$

These coordinates may also be related to the hyberbolic coordinates $\psi$ and $\phi$

$$
\psi=4^{-1}\left(x^{2}-y^{2}\right),
$$




$$
\begin{aligned}
& \phi=2^{-1} x y, \\
& \psi^{2}=16\left[\left(\psi-\frac{a^{2}}{4}\right)^{2}+\phi^{2}\right], \\
& \tan \Phi=\phi\left(\psi-\frac{a^{2}}{4}\right)^{-1}, \\
& \phi=4^{-1} \Psi \sin \Phi, \\
& \psi=4^{-1}\left(a^{2}+\Psi \cos \Phi\right)
\end{aligned}
$$

Equation (A3) may be derived easily using Eqs. (AI0), (All), and the following fact which may easily be derived from Eqs. (A6) and (A7).

$$
d s^{2}=\left(\psi^{2}+\phi^{2}\right)^{-1 / 2}\left(\mathrm{~d} \psi^{2}+\mathrm{d} \phi^{2}\right) .
$$




\section{REFERENCES}

$I_{\mathrm{P}}$. A. Sweet, in Electromagentic Phenomena in Cosmical Physics (I.A.U. Syposium No. 6), (Cambridge University Press, Cambridge, England, 1958), p. 123.

2E. N. Parker, J. Geophys, Res. $6 \underline{2}, 609$ (1957).

${ }^{3}$ H. E. Petschek, in The Physics of Solar Flares - AAS-NASA Symposium, ED. by W. N. Hess, (NASA SP-SO, 1964), p. 425.

${ }^{4}$ H. P. Furth, J. Killeen, and M. N. Rosenbluth, Phys. Fluids 6,459 (1963).

5. V. Waddell, M. N. Rosenbluth, D. A. Monticello, and R. B. White, Nucl. Fusion Lett. 16, 528 (1976).

${ }^{6}$ V. M. Vasyliunas, Rev. of Geophys, and Space Sri. 13, 303 (1975). 


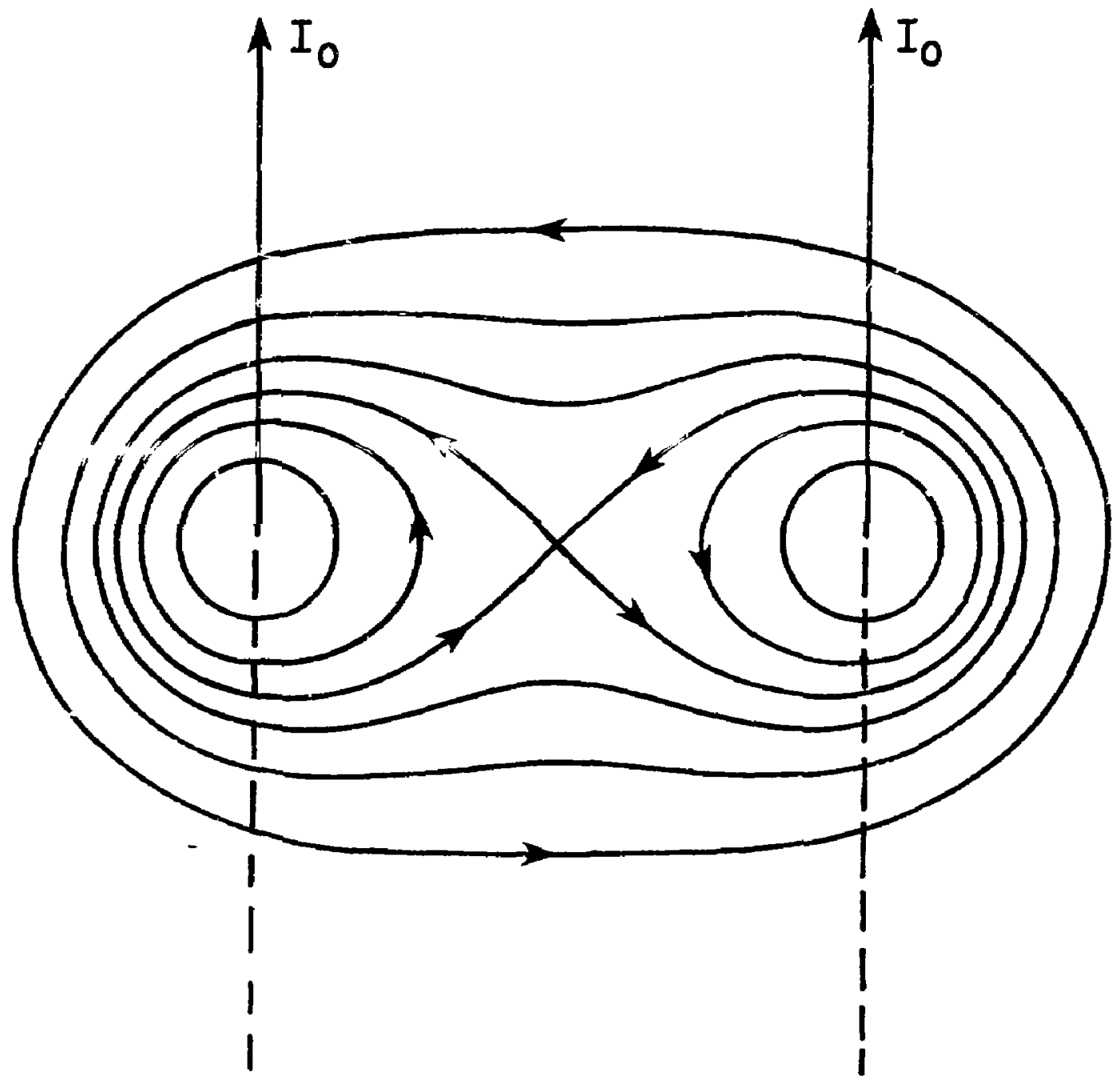

Fig. 1: Initial configuration of the wires and the magnetic field. 


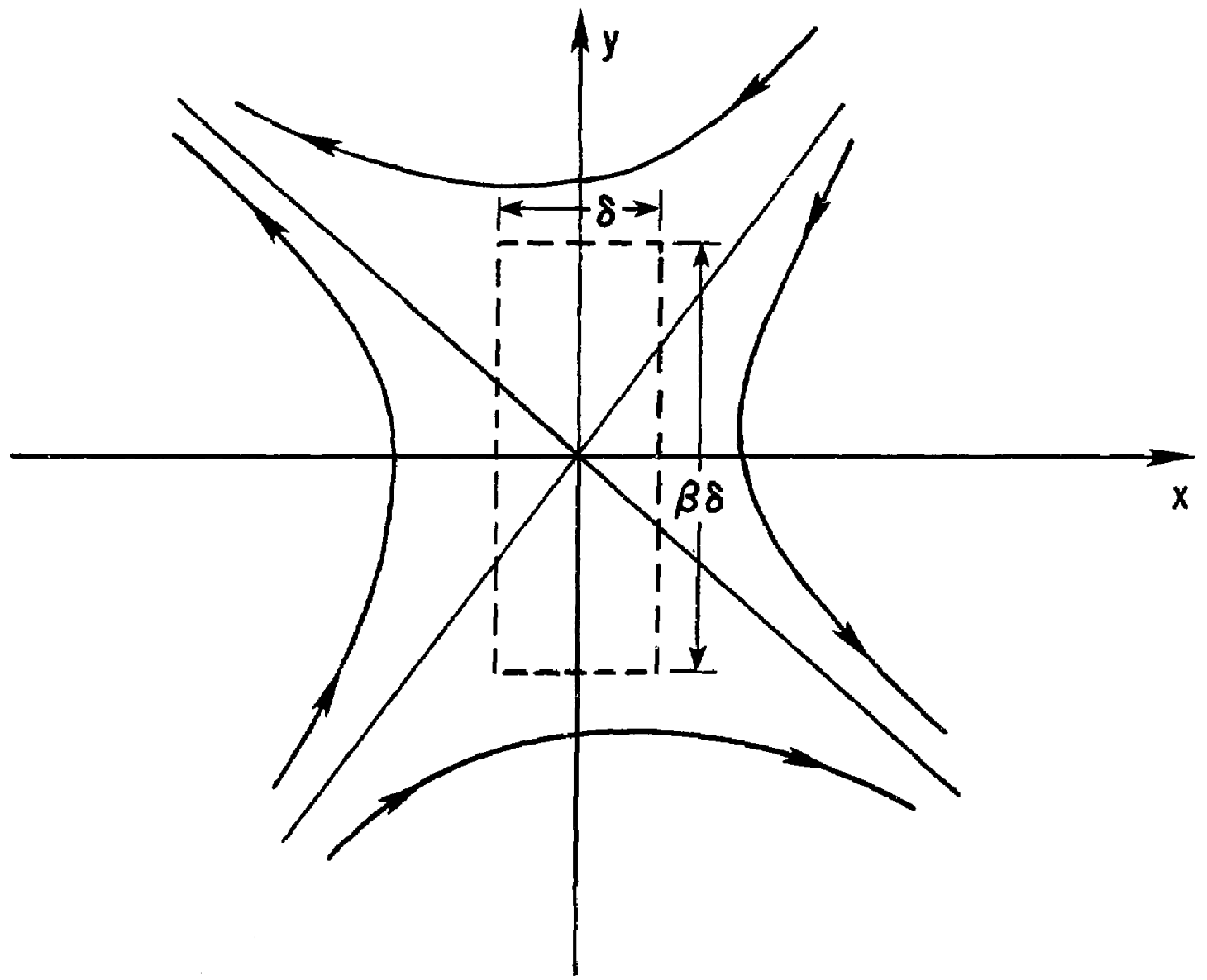

Fig. 2: Schematic view of the inner region. 


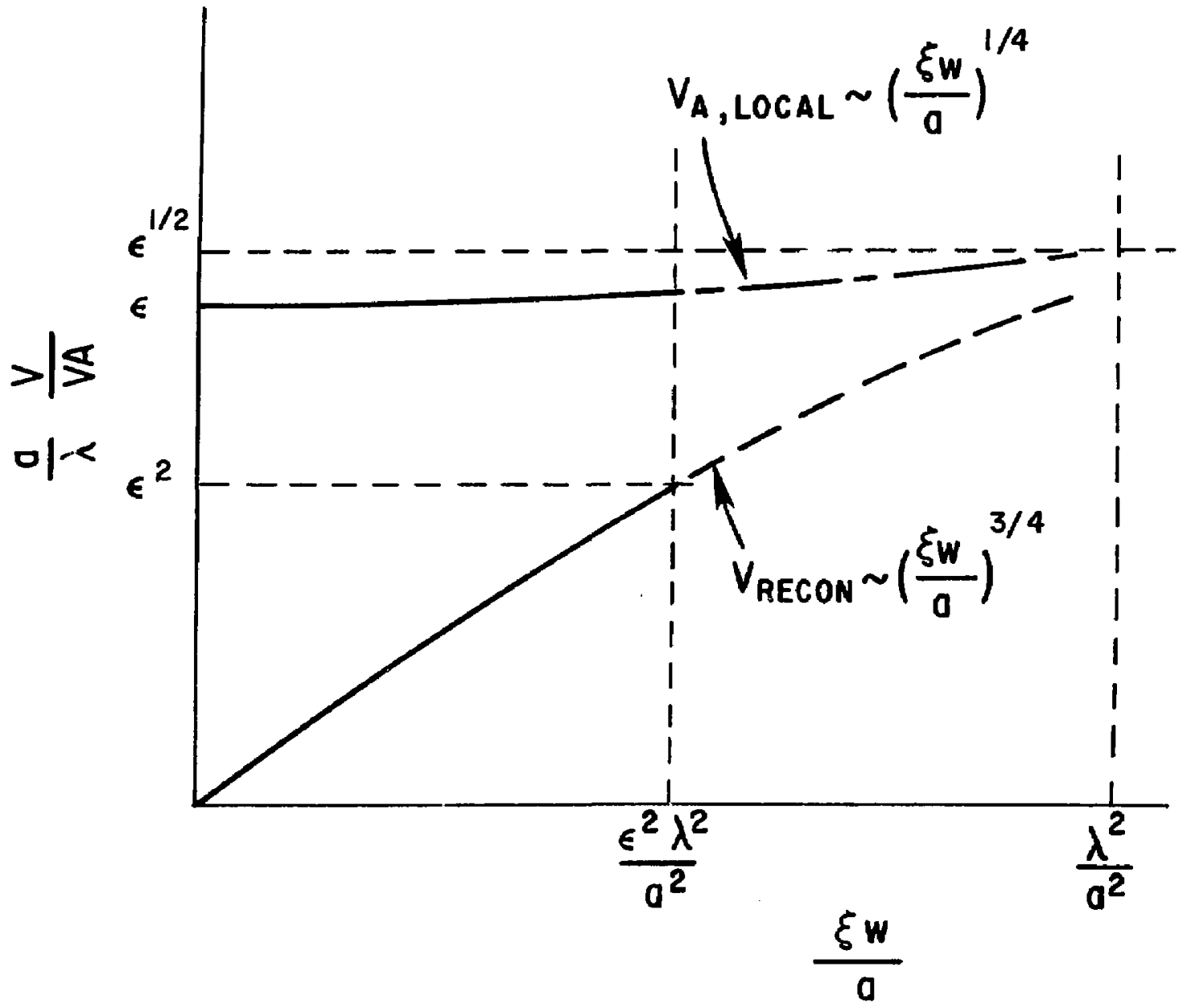

782030

Fig. 3: Effective reconnection rate vs wire displacement. 


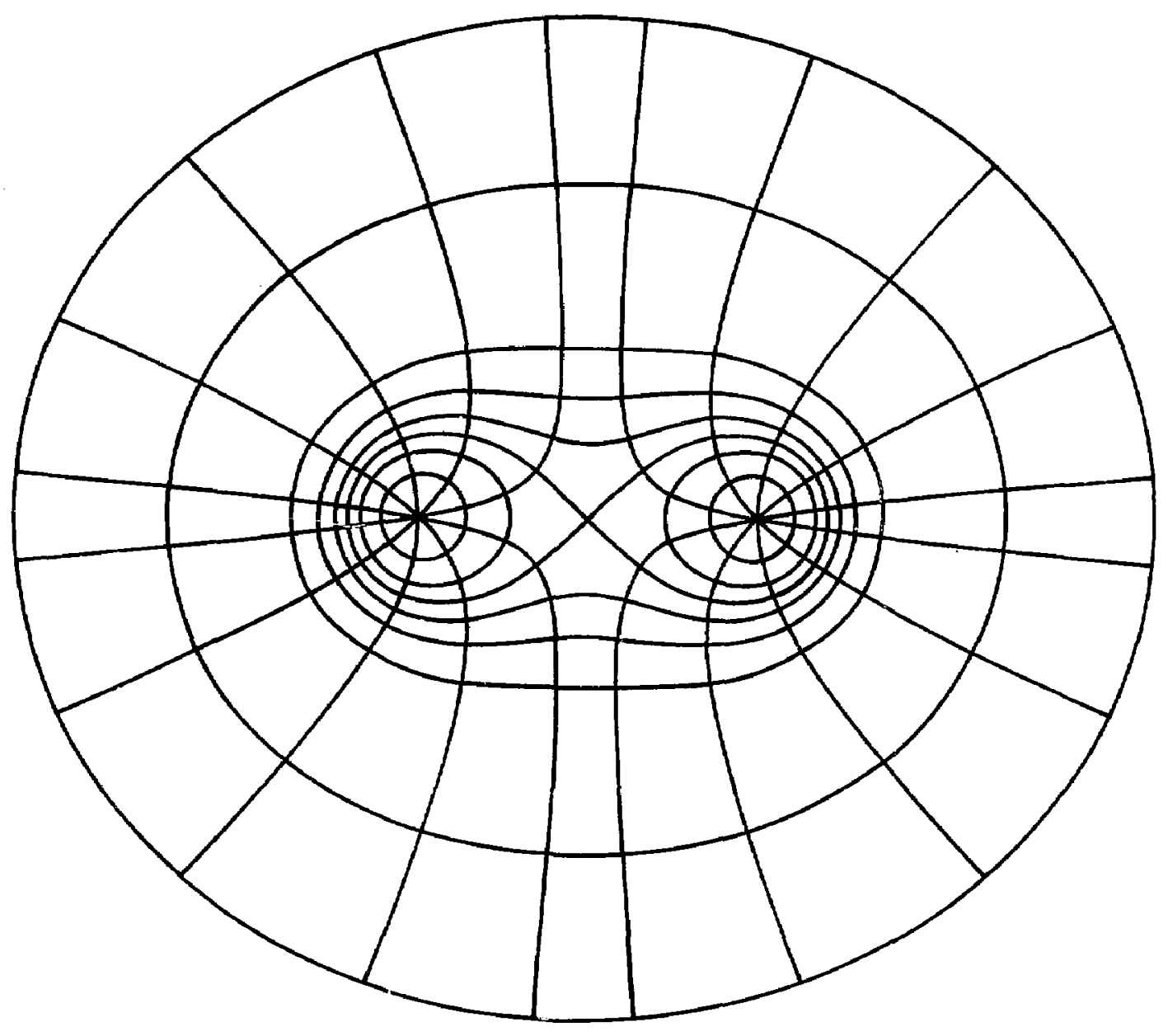

782029

Fig. 4: $\Psi, \Phi$, coordinate system. 